\title{
Uma metodologia de custo zero para medida de atrito de rolamento em VANTs
}

\author{
A zero cost methodology for the measurement of the rolling resistance in UAVs
}

\author{
C.C. Pellegrini* ${ }^{*}$, V.R. Alves
}

${ }^{1}$ Universidade Federal de São João del-Rei, Departamento de Ciências Térmicas e dos Fluidos, São João del-Rei, MG, Brasil

Recebido em 21 de novembro de 2019. Revisado em 27 de abril de 2020. Aceito em 30 de maio de 2020.

\begin{abstract}
A despeito do fato que medidas do coeficiente de atrito de rolamento são importantes em diversas áreas, o assunto em geral não é adequadamente tratado no ensino médio ou superior. Tal situação priva o estudante do conhecimento dos recursos experimentais utilizados para medir o coeficiente de atrito, além de comprometer o entendimento do fenômeno. Além disso, certos pares de materiais de interesse para a engenharia simplesmente não são encontrados na literatura, como no caso dos pneus utilizados em veículos aéreos não tripulados (VANTs). $\mathrm{Na}$ tentativa de suprir ambas as lacunas, o presente trabalho apresenta uma metodologia para a determinação do coeficiente de atrito de rolamento em VANTs. O método empregado é extremamente simples, em termos de implementação e da teoria utilizada, mas apresenta interesse do ponto de vista didático e devido ao fato de seu custo ser praticamente zero, utilizando apenas um plano inclinado e medidas da distância percorrida pelo VANT. Os resultados mostram valores do coeficiente de atrito dependentes do peso do veículo. Os valores obtidos são discutidos e sugestões para o aprimoramento futuro do experimento são apresentadas.
\end{abstract}

Palavras-chave: atrito de rolamento, experimento de baixo custo, VANT.

Despite the fact that measurements of the rolling friction coefficient are important in several areas, the subject in general is not adequately addressed in high school or college. This situation deprives the student of the knowledge of the experimental resources used to measure the friction coefficient, in addition to compromising the understanding of the phenomenon. In addition, certain pairs of materials of interest to engineering are simply not found in the literature, as in the case of tires used in unmanned aerial vehicles (UAVs). In an attempt to fill both gaps, the present work presents a methodology for determining the rolling friction coefficient in UAVs. The method used is extremely simple, in terms of its implementation and of the theory used, but it is still interesting from a didactic point of view and due to the fact that its cost is practically zero, using only an inclined plane and measures of the distance covered by the UAV. The results show friction coefficient values depending on the vehicle's weight. The values obtained are discussed and suggestions for the future improvement of the experiment are presented.

Keywords: rolling resistance, low cost experiment, UAV.

\section{Introdução}

Ainda que medidas do coeficiente de atrito de rolamento, conhecido como $\mu_{R}$, sejam importantes em inúmeras áreas do conhecimento, o assunto em geral não é adequadamente abordado no ensino médio ou no ensino superior. A frase "Desprezando-se os atritos de rolamento"é ouvida com quase tanta frequência quanta a frase "Desprezandose a resistência do ar". Tal situação, já percebida por diversos autores ( 1] por exemplo), faz mais do que privar o estudante do conhecimento dos recursos experimentais utilizados para medir o coeficiente; acaba privando-o do próprio entendimento do fenômeno.

Felizmente, alguns artigos recentes de caráter didático abordaram o problema em detalhes. Por exemplo, [2] desenvolve soluções analíticas para o rolamento de um objeto sobre uma superfície horizontal plana, enquanto 1 .

*Endereço de correspondência: pelle@ufsj.edu.br detalha o modelo teórico usado para descrever o fenômeno e propõe um experimento de baixo custo para a medida de $\mu_{R}$. Já [3] descreve o experimento montado para medir o atrito de rolamento em um veículo de elevada eficiência energética, destinado a participar da Shell Eco-Marathon, uma competição estudantil anual organizada pela Shell Europe. Mesmo o fenômeno do atrito de escorregamento mostra facetas pouco exploradas, como fica evidente no estudo de 4], que chama a atenção para o deslocamento lateral da força normal na presença da força de atrito.

Uma aplicação interessante e que demonstra a importância de se estudar o atrito de rolamento é a decolagem de aeronaves. Em 1999 a Sociedade de Engenheiros da Mobilidade (SAE Brasil) criou a competição AeroDesign, com o objetivo de propiciar "a difusão e o intercâmbio de técnicas e conhecimentos de Engenharia Aeronáutica, através de aplicações práticas e da competição entre equipes". Nesta competição, estudantes devem formar 
equipes, sob a tutela de um orientador acadêmico, para conceber, projetar e construir um veículo aéreo não tripulado (VANT) cargueiro destinado a cumprir desafios que variam a cada edição do certame. Os projetos são anualmente postos à prova em uma competição de âmbito internacional realizada nas instalações do Departamento de Ciência e Tecnologia Aeroespacial, em São José dos Campos, SP.

Neste contexto, o assunto do atrito de rolamento foi abordado em um estudo recente sobre a dinâmica da decolagem com forças dependentes da velocidade por [5]. O trabalho propôs soluções analíticas para o cálculo das distâncias de pouso e decolagem de aeronaves e comparou, com sucesso, os resultados com dados obtidos na decolagem de uma aeronave comercial leve e do VANT desenvolvido pelos autores para a competição AeroDesign de 2014.

Durante os anos que se seguiram à proposta da solução, os autores seguidamente a utilizaram para estimar o desempenho de outros VANTs desenvolvidos pelo grupo. Ficou claro que a correta estimativa de $\mu_{R}$ era fundamental para o sucesso dos cálculos. Como relatado por um dos envolvidos no projeto, 'Era possível obter qualquer distância de decolagem e de pouso manipulando adequadamente o valor do coeficiente". Esse problema mostrou-se crítico no projeto de 2019, onde duas cargas com características bastante distintas precisariam ser transportadas: uma leve e volumosa e outra pesada e pequena. Ficou claro também que os valores de $\mu_{R}$ relatados na literatura, típicos de pneus de aviação ou automotivos, eram inadequados à realidade dos VANTs projetados.

O presente trabalho aparece como uma tentativa de suprir a lacuna mencionada, propondo uma metodologia para a determinação mais precisa de $\mu_{R}$ para os VANTs construídos pelo grupo. O método empregado é extremamente simples, em termos de implementação e da teoria utilizada, mas apresenta interesse devido ao seu custo zero, um requisito cada vez mais importante em tempos de cortes orçamentários. O presente trabalho se propõe também a explicar porque os valores de $\mu_{R}$ extraídos da literatura não representam adequadamente o fenômeno para as aeronaves construídas, oferecendo uma base teórica mais sólida a ser utilizada pela Equipe em projetos futuros.

Cumpre mencionar que a ênfase no baixo custo não é tão recente quanto possa parecer. Alguns estudos abordando montagens experimentais de baixo custo destinadas a medir os coeficientes de atrito de escorregamento foram propostas por [6], 7] e [8 em 2002, 2007 e 2012 respectivamente.

\section{Análise do Problema}

Segundo o princípio da conservação da energia, a variação da energia mecânica total de um corpo que se desloca sobre uma superfície e está sujeito a forças não conservativas de atrito é igual ao trabalho realizado por estas forças, ou seja,

$$
\Delta E_{m e c}=W_{\text {dissip }}
$$

Em um sistema mecânico em que a força de atrito de rolamento predomina sobre outras forças dissipativas, este princípio pode ser utilizado para medir o coeficiente de atrito da seguinte maneira. Suponha que o corpo seja colocado no alto de uma rampa e deixado livre para se deslocar em rolamento puro, sob ação do peso próprio $W$, em $t \geq t_{0}$ (Figura 1).

Sobre o veículo agem, durante a descida e até o repouso, as forças de atrito de rolamento em todas as rodas, sendo o trabalho associado a estas forças, $W_{R}$. Agem também as forças de atrito nos mancais, rolamentos e outros elementos de fixação das rodas à estrutura, inclusive a suspensão, caso haja (com trabalho $W_{M}$ ). Agem ainda a força de arrasto aerodinâmico (com trabalho $W_{A}$ ) e outras forças parasitas (com trabalho $W_{O}$ ). Deste modo,

$$
\Delta E_{m e c}=W_{R}+W_{M}+W_{A}+W_{O}
$$

Algumas considerações sobre estas forças e seus trabalhos associados são apresentadas a seguir.

As forças relevante para o cálculo do atrito de rolamento estão principalmente relacionadas a dois fatores: (i) à conversão de energia cinética em energia térmica, que ocorre na deformação cíclica das rodas e (ii) à adesão entre as rodas e a superfície ( [9] $)$, ou seja,

$$
P=N+F_{s}
$$

em que $P$ é a força total de contato, $N$ é a força normal e $F_{s}$ a força de adesão. Em geral $F_{s} \ll N$, de modo que $F_{s}$ é desprezada na maioria dos estudos e $P$ é tomada simplesmente como sendo igual a $N$. Este conceito irá se mostrar bastante relevante e será investigado mais profundamente numa seção posterior do presente trabalho.

Todas as outras forças relevantes estão incluídas nos termos $W_{M}, W_{A}$ e $W_{O}$. Um abrangente estudo sobre o atrito de rolamento pode ser encontrado em [10] Cap. 3, e apresenta detalhes sobre tais forças. Apresentaremos a seguir algumas considerações sobre as forças parasitas.

Dentre as forças embutidas no termo $W_{O}$, ocorrem frequentemente em projetos de VANTs forças devidas ao desalinhamento das rodas com a direção do movimento

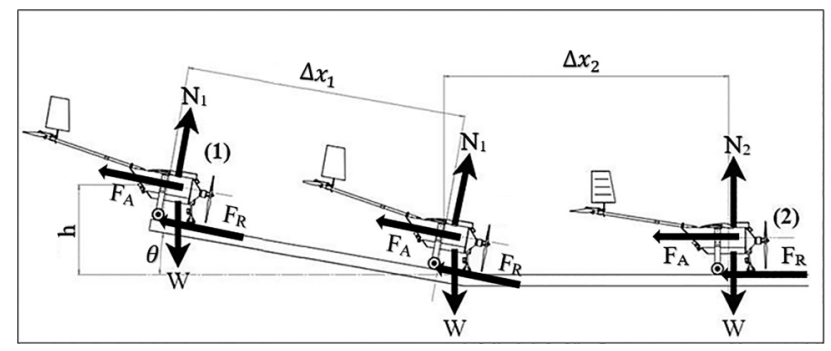

Figura 1: Esquema da montagem experimental. 
e forças devidas a irregularidades na pista. Tais irregularidades diferem da rugosidade da pista, que é modelada pelo atrito de rolament ${ }^{1}$ Na presente análise as perdas associadas a $W_{O}$ também incluem buracos nas pista, a presença de pequenas pedras soltas e areia, e a transição entre a parte inclinada e a parte plana da rampa de testes. Incluem-se também forças devidas a danos sofridos pelos pneus, forças originadas na vibração de partes da aeronave (que em geral não possui suspensão) e outras. Naturalmente, é extremamente importante minimizar estas ocorrências durante o projeto do experimento. Ainda mais importante é minimizá-las durante a construção e o uso do VANT.

Entretanto, não é tarefa simples modelar cada uma das parcelas de trabalho descritas. Como no presente estudo apenas o total de tais parcelas nos interessa, $W_{M}$, $W_{A}$ e $W_{O}$ serão absorvidas por $W_{R}$. Isto, por si só, não constitui uma aproximação; a verdadeira aproximação neste ponto será considerar que o trabalho total das forças dissipativas pode ser modelado da mesma forma que o trabalho do atrito de rolamento, ou seja, como

$$
W_{\text {dissip }} \approx \mu_{R} P \Delta x
$$

em que $P$ é a força de contato dada pela Eq. $3 . \Delta x$ é a distância percorrida e $\mu_{R}$ é um coeficiente de atrito de rolamento efetivo, que absorveu os outros efeitos dissipativos. Esta hipótese, tradicional na literatura, é razoável em sistemas em que predomine a força de atrito de rolamento, um fato que pode ser verificado quantitativamente.

A Figura 2 mostra as forças atuantes durante a decolagem do VANT construído para a competição de 2019. A velocidade foi variada entre 0 e $10,4 \mathrm{~m} / \mathrm{s}$, limite em que a aeronave decola e, portanto a força de sustentação iguala-se ao peso. Neste instante, a normal torna-se zero e a força de atrito de rolamento anula-se, como mostra a Equação 4 .

A Figura 3 mostra em detalhes a relaçao entre as forças de arrasto e de atrito de rolamento, evidenciando que

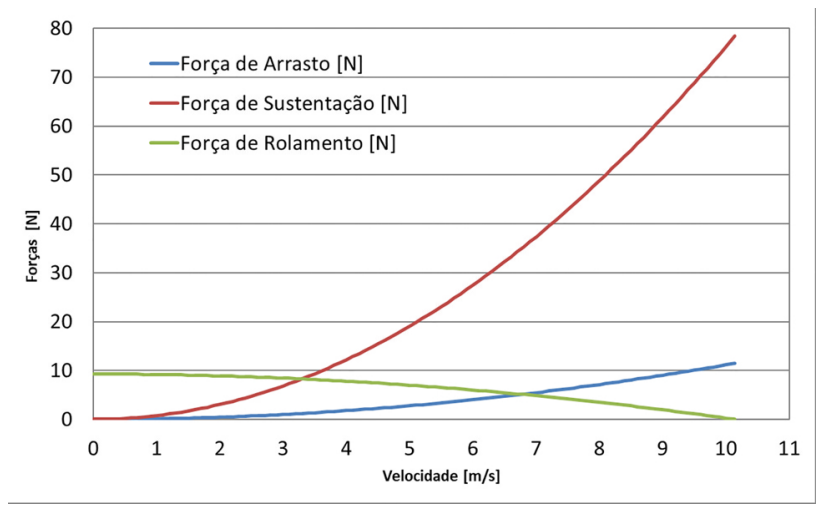

Figura 2: Forças atuantes durante a decolagem.

\footnotetext{
$\overline{1}^{1}$ a realidade, uma ampla gama de escalas de comprimimento está envolvida na interação entre as rodas e a superfície. Na prática, porém, apenas duas escalas de comprimento são usadas: a da textura (ou rugosidade) da pista e a dos defeitos da pista
}

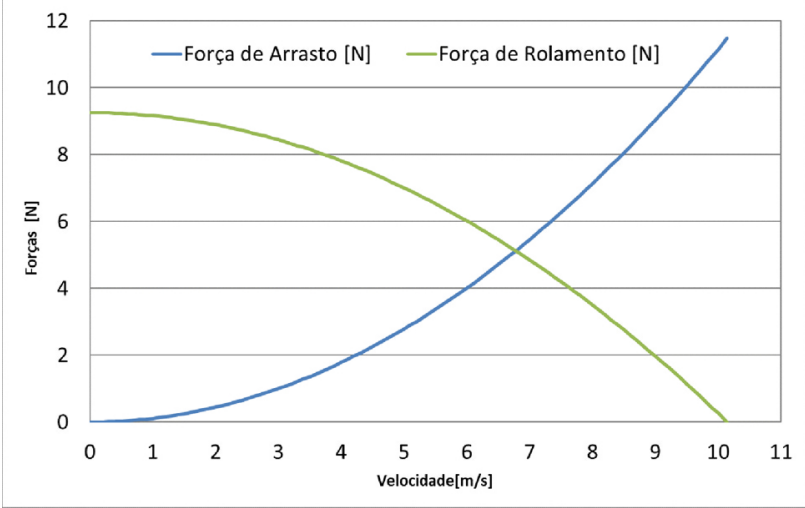

Figura 3: Forças dissipativas atuantes durante a decolagem.

para manter a desejada relação $F_{R} \gg F_{A}$ é preciso ter $V \leq 2,0 \mathrm{~m} / \mathrm{s}$. Para garantir isto, basta escolher uma rampa com inclinação adequadamente pequena.

Caso a relação $F_{R} \gg F_{A}$ não se verifique, a aproximação usada permanece matematicamente válida, mas $\mu_{R}$ passará a depender da velocidade do corpo, uma vez que a força de arrasto aerodinâmico também depende. Além disso, fica claro que valores de $\mu_{R}$ obtidos da literatura para pneus de grande porte podem não representar adequadamente o atrito nos VANTs, justamente devido ao fato de havermos embutido em $\mu_{R}$ os efeitos de $W_{M}, W_{A}$ e $W_{O}$.

Considerando-se, então, as partes inclinada (1) e horizontal (2) do experimento, e supondo por hora que $F_{s} \ll N$, segue-se que

$$
\Delta E_{m e c}=\mu_{R}\left(N_{1} \Delta x_{1}+N_{2} \Delta x_{2}\right)
$$

Entre os pontos em que o corpo foi liberado do repouso e o repouso subsequente ao rolamento, $\Delta E_{m e c}=m g h$. Portanto,

$$
\mu_{R}=\frac{m g h}{N_{1} \Delta x_{1}+N_{2} \Delta x_{2}}
$$

No trecho (2) a força normal iguala-se ao peso do VANT, ou seja, $N_{2}=m g$, enquanto no trecho (1), $N_{1}=$ $m g \cos \theta$, de modo que

$$
\mu_{R}=\frac{h}{\cos \theta \Delta x_{1}+\Delta x_{2}}
$$

A rigor, ao longo do trajeto tem-se

$$
\begin{aligned}
& N_{1}=W \cos \theta-F_{S}, \\
& N_{2}=W-F_{S}
\end{aligned}
$$

nos trechos inclinado e horizontal, respectivamente, sendo $F_{S}=(1 / 2) \rho C_{S} S V^{2}$ a força de sustentação gerada pelas asas, em que $\rho$ é a massa específica do ar, $C_{S}$ é o coeficiente de sustentação, $S$ é a superfície da asa e $V$ é a velocidade do VANT 11].

Contudo, se tal consideração for feita, será necessário incluir a força de arrasto aerodinâmico dentre as forças dissipativas consideradas, de modo a manter a coerência 
teórica. Com isso, a Equação 2 perderia seu caráter global, tornando-se uma EDO para $V=V(x)$.

Para manter a simplicidade da análise, deve-se portanto escolher um ângulo $\theta$ que garanta que a velocidade de rolamento seja pequena o suficiente para que $F_{S}$ seja muito menor que $F_{R}$, mas grande o suficiente para que os erros na medida dos deslocamentos não afetem significativamente os resultados. Pode-se verificar na Figura 2 que a relação $F_{S} \ll F_{R}$ vale para $V \leq 1,5 \mathrm{~m} / \mathrm{s}$.

Não é possível calcular a velocidade máxima durante o trajeto a priori, de forma a comprovar que tal hipótese seja válida. Para isso seria preciso integrar a equação do movimento e realizar os cálculos que, evidentemente, dependeriam do valor de $\mu_{R}$, que é justamente o que se deseja medir. Uma solução paliativa para o problema seria utilizar a velocidade média do deslocamento como estimativa. Isto será feito na próxima seção que mostra a construção do experimento

\section{Procedimento experimental}

Para execução experimental do estudo, uma aeronave montada com diferentes configurações de carga foi posicionada sobre uma rampa asfaltada (na verdade uma parte do estacionamento do Campus, Figura 4). Verificou-se que o asfalto possuía características semelhantes ao da pista usada na competição, pequena inclinação, $\theta=10$, e mediu-se $\Delta x_{1}=1,30 \mathrm{~m}$.

Vale lembrar que os pneus utilizados no experimento, assim como na Competição SAE Brasil AeroDesign e, em geral, em aeromodelismo, são fabricados em borracha maciça ou em espuma de borracha e não há valores do coeficiente de atrito de rolamento disponíveis na literatura para estes materiais.

A escolha do local condicionou-se também ao fato de que a transição entre a parte inclinada e a parte plana da rampa não fosse abrupta, minimizando a conversão de energia cinética em trabalho de deformação dos pneus durante o impacto, embutidas no termo $W_{O}$ da Equação 2 .

Utilizando uma mangueira em forma de U parcialmente preenchida com água para estabelecer o plano horizonta $2^{2}$,

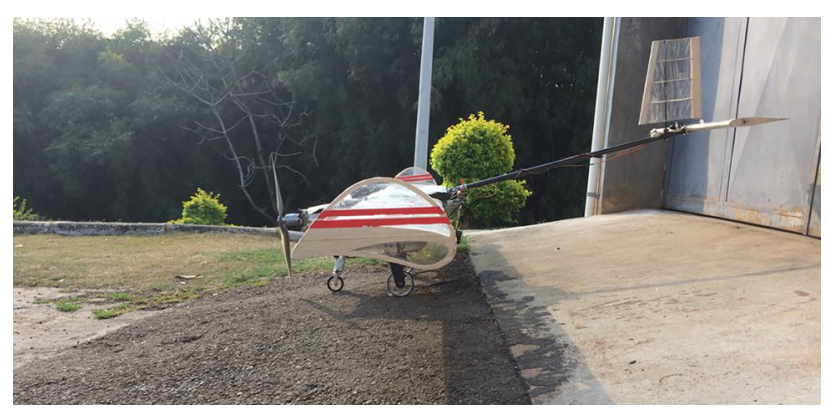

Figura 4: Montagem experimental.

2O conhecido "nível de pedreiro" mediu-se uma distância vertical $h=0,44$ m entre o CG da aeronave posicionada no topo e na base da rampa.

Cada experimento consistiu em posicionar o VANT em um ponto fixo, liberá-lo do repouso e medir a segunda parte da distância percorrida até o repouso, $\Delta x_{2}$. A Equação (7) foi então utilizada para o cálculo de $\mu_{R}$. O resultado das medidas realizadas e os pesos das quatro configurações de carga escolhidas para o experimento são apresentados na Tabela 1. Foram tomadas dez medidas de distância para cada carga. $\mathrm{O}$ valores escolhidos para o peso são típicos da competição, variando entre 2 e 8 kgf, sendo que valores acima de $8 \mathrm{kgf}$ estão fora dos limites de projeto do aeronave.

A Tabela 1 mostra que os descolamentos médios, $\Delta \bar{x}_{2}$, variam entre 2,03 e 2,67 m conforme a carga, com desvios padrão, $\sigma$, da ordem de $0,1 \mathrm{~m}$. As variações observadas durante as medidas são atribuídas a fatores incontroláveis no experimento, como irregularidades na pista. Os valores de $\mu_{R}$ obtidos situam-se em torno de 0,1 . Como previsto, tais valores diferem por quase uma ordem de grandeza dos obtidos da literatura para pneus de grande porte rolando em pistas asfaltadas, tipicamente localizados no intervalo 0,02-0,03 [12]. Valores da ordem de 0,1 para pneus aeronáuticos são encontrados no rolamento de sobre pistas de grama alta.

Durante o experimento, o deslocamento total do VANT $\left(\Delta x_{1}+\Delta x_{2}\right)$ foi sempre igual ou inferior a $4,1 \mathrm{~m}$, percorridos em um tempo (cronometrado durante o experimento) em torno de 4 segundos. Isso nos fornece uma velocidade média menor ou igual a $1,0 \mathrm{~m} / \mathrm{s}$, o que valida a hipótese de que as forças de sustentação podem ser desconsideradas, ao menos na maior parte do trajeto. A Figura 2 atesta este fato, sugerindo um valor da razão $F_{R} / F_{S} \approx 12$.

A Figura 5 mostra a dependência de $\mu_{R}$ com o peso total do VANT. Pode-se perceber uma relação linear entre as duas grandezas, com $\mu_{R}$ diminuindo à medida que o peso aumenta. Um ajuste de curva linear entre os limites testados fornece

$$
\mu_{R}=0,14-0,0036 W
$$

com coeficiente de determinação $R^{2}=0,9997$. Esta conclusão é bastante interessante e merece um aprofundamento, a ser apresentado na próxima seção.

Tabela 1: Valores experimentais do deslocamento horizontal e do coeficiente de atrito de rolamento. Pneus de espuma sobre pista asfaltada.

\begin{tabular}{lccc}
\hline $\mathrm{W}[\mathrm{N}]$ & $\Delta \bar{x}_{2}[\mathrm{~m}]$ & $\sigma[\mathrm{m}]$ & $\mu_{R}$ \\
\hline 19,66 & 2,03 & 0,090 & 0,133 \\
40,06 & 2,22 & 0,081 & 0,126 \\
55,35 & 2,38 & 0,105 & 0,120 \\
78,45 & 2,67 & 0,131 & 0,112 \\
\hline
\end{tabular}




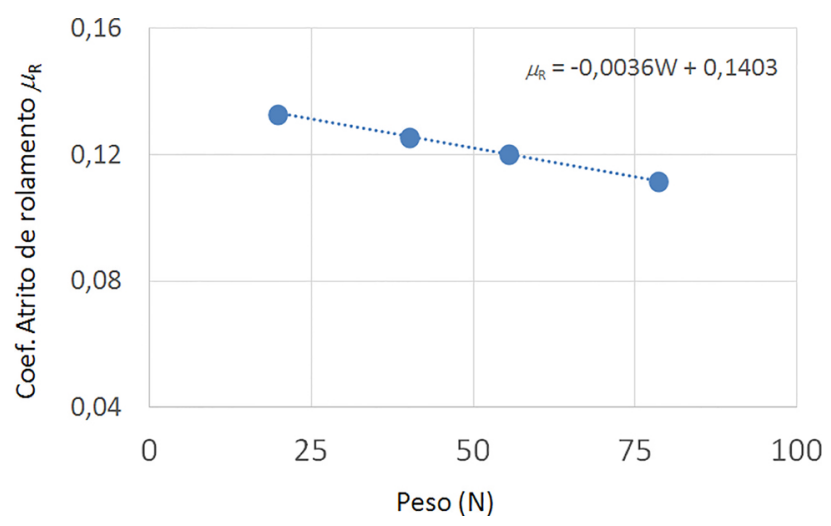

Figura 5: Relação entre o coeficiente de atrito de rolamento e o peso total do VANT.

\section{Análise dos resultados}

Segundo [13], o coeficiente de atrito de rolamento aumenta com o aumento do peso para pneumáticos com pressão interna mantida constante. Dados obtidos por [14] mostram que a seguinte curva empírica é capaz de representar bem a força de atrito de rolamento em uma ampla gama de pesos, pressões de inflamento e velocidades, para diversos tipos de pneus:

$$
P=A p^{\alpha} M^{\beta}\left(a+b V+c V^{2}\right)
$$

em que $p$ é a pressão de inflamento, $M$ é a massa associada ao peso sobre o pneu, $V$ é a velocidade do veículo e $A$, $a, b, c, \alpha$ e $\beta$ são constantes. Os experimentos mostraram que, para os pneus radiais modernos, $-0,5 \leq \alpha \leq-0,3$, indicando que $F_{R}$ (e portanto $\mu_{R}$ ) diminui com o aumento da pressão, como seria de se esperar.

Por outro lado, $0,8 \leq \beta \leq 1,1$, indicando um aumento quase linear de $\mu_{R}$ com o peso. O motivo é o aumento da deformação do pneu e o consequente aumento na energia convertida em calor durante o processo. Ao início do presente experimento, este era o comportamento esperado para os pneus de espuma de borracha utilizados, mas isso não ocorreu.

Verificou-se que a literatura mostra valores de $\mu_{R}$ diminuindo com o aumento do peso para o contato entre superfícies rígidas, como rodas de aço sobre trilhos de aço, em linhas férreas por exemplo. Ao longo dos anos, uma diversidade de explicações para este comportamento contra-intuitivo foi apresentada. A explicação atualmente aceita (devida a [9]) atribui este comportamento à força de adesão entre as superfícies, que se torna maior sob cargas pequenas. Naturalmente, este comportamento só se torna evidente sob cargas totais pequenas, quando a força normal é pequena também.

A Figura 5 deixa claro que, a despeito da diferença entre os materiais utilizados, o comportamento observado em nosso experimento assemelha-se a este. Os motivos para isto não são, no momento, totalmente claros posto que as rodas analisadas não são constituídas de material rígido. Isso nos leva a propor algumas hipóteses.
Foi observado anteriormente, no âmbito da Eq. 3, que o contato roda-superfície inclui tanto efeitos de deformação quanto de adesão. Acompanhando a maior parte da literatura utilizada em cursos de Física e Engenharia, foi então suposto que $F_{s} \ll N$ e $P$ foi substituído por $N$ em toda a análise subsequente. Porém, a literatura mais específica, em particular 9], mostrou que nem sempre os efeitos de adesão são desprezíveis. De fato, para explicar o comportamento do contato entre materiais rígidos é necessário considerar que os efeitos das forças de adesão sejam predominantes.

Fica, então, claro que atuam no fenômeno do atrito de rolamento ao menos dois efeitos distintos e com influências opostas sobre a força total $P$ : (i) o efeito de deformação das rodas, que aumenta $\mu_{R}$ à medida que o peso sobre a roda aumenta, e (ii) o efeito de adesão, que diminui $\mu_{R}$ à medida que o peso sobre a roda aumenta. Evidentemente, a resposta final do par roda-superfície à variação da carga aplicada será um balanço de qual efeito é preponderante no caso considerado.

Comparando os resultados experimentais obtidos por 9], mostrado na Fig. 6. com os nossos, expressos na Fig. 5 percebe-se uma semelhança bastante acentuada na região à direita do gráfico.

Segue-se, portanto, a conclusão de que o material utilizado em nosso experimento apresenta efeitos de adesão que predominam sobre os de deformação, ainda que isso não seja inicialmente óbvio. A Figura 7, que mostra a deformaçao das rodas utilizadas sob carga, de fato sugere o contrário.

Uma explicação possível seria de que os efeitos de adesão, bastante difíceis de estimar, possam ser ainda maiores que os de deformação, dada a natureza "grudenta"da espuma de borracha. Este fato não teria sido detectado em estudos anteriores simplesmente porque nunca havia existido interesse em realizar experimentos com rodas de espuma.

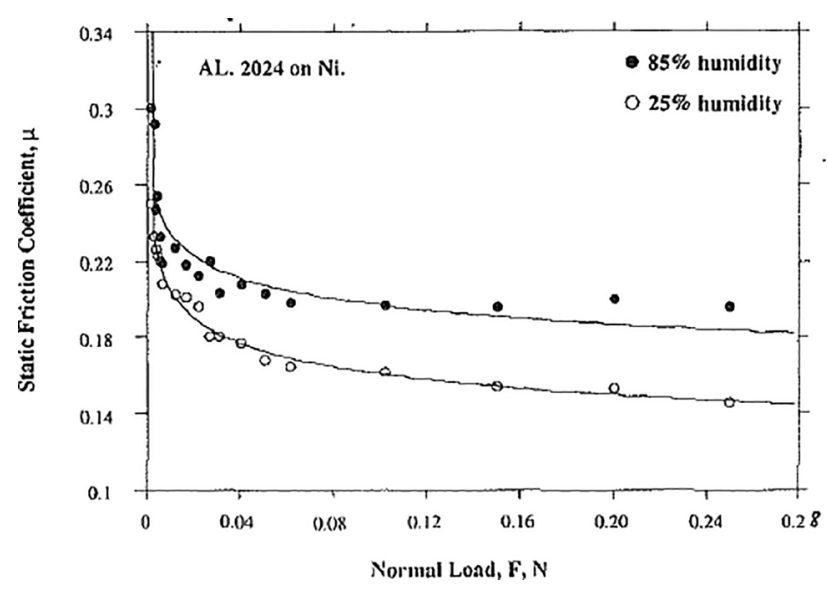

Figura 6: Efeito da carga normal sobre o coeficente de atrito de rolamento. Alumínio 7075 sobre Níquel. Reproduzido diretamente de [9] 


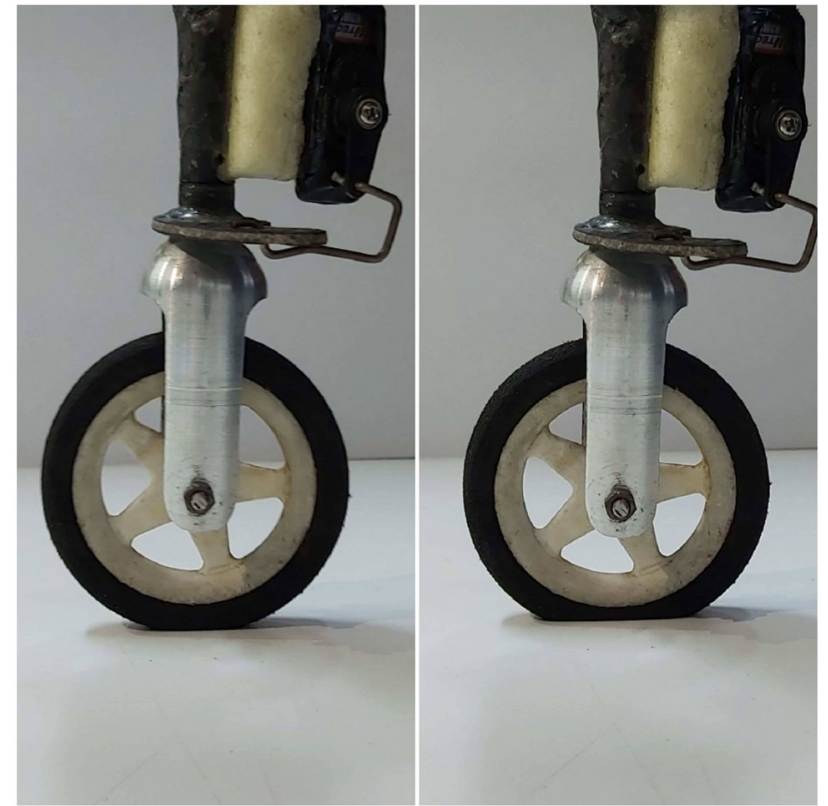

Figura 7: Deformação variável das rodas conforme a carga. Exagerada para melhor compreensão.

\section{Conclusões}

Neste trabalho, uma metodologia de custo zero foi proposta para a medida do atrito de rolamento no trem de pouso de um VANT. O resultado é relevante tanto para estimar o desempenho deste tipo de aeronave durante o pouso e a decolagem, quanto para esclarecer detalhes teóricos relevantes a respeito do atrito de rolamento. Entre eles destacam-se: (i) a diferença entre os valores de $\mu_{R}$ obtidos no experimento e os disponíveis na literatura; (ii) a dependência de $\mu_{R}$ com o peso da aeronave; (iii) quais os efeitos considerados e desprezados durante a análise.

$O$ fato dos valores experimentais de $\mu_{R}$ diferirem dos valores encontrados na literatura, deve-se a dois motivos. Em primeiro lugar, evidentemente não seria de se esperar os mesmos valores, uma vez que os pneus típicos dos VANTS são confeccionados em borracha maciça e espuma de borracha. Isso não torna o presente estudo menos importante, uma vez que tais valores não são encontrados na literatura e são fundamentais para um projeto de sucessd 3

Além disso, na modelagem matemática adotada, os efeitos de $W_{M}, W_{A}$ e $W_{O}$ foram embutidos em $\mu_{R}$, conforme discutido na seção Análise do Problema. Em particular, é interessante observar que efeitos geralmente desprezíveis em veículos de grande porte, como danos sofridos aos pneus ou a presença de areia e pequenas pedras na pista, podem ser relevantes aqui.

Em relação à dependência de $\mu_{R}$ com o peso da aeronave, deve-se notar que ainda que a literatura não especializada sugira considerar $\mu_{R}$ como uma constante

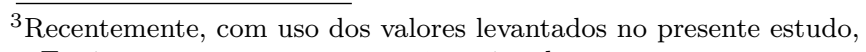
a Equipe sagrou-se tetra campeã nacional por simplicidade, este coeficiente indiscutivelmente depende de diversos fatores, mais ou menos importantes conforme o caso considerado. No presente estudo, uma dependência muito clara entre $\mu_{R}$ e $W$ foi observada para os carregamentos testados. O comportamento inesperado observado, isto é, a redução de $\mu_{R}$ com o aumento de $W$, não pôde ser explicado de maneira conclusiva e será o objeto de investigação posteriores, pois descortina a possibilidade de se minimizar o atrito de rolamento, melhorando o desempenho dos VANTs estudados, no momento (crucial) de sua decolagem.

Em relação aos efeitos físicos desconsiderados, acreditamos ser este um debate bastaste relevante na análise de qualquer fenômeno físico, uma vez que é quase sempre impossível realizá-la sem o uso de hipóteses simplificadoras. Contudo, os estudantes frequentemente perdem o controle das hipóteses empregadas e terminam por utilizar a teoria fora dos seus limites de validade. Exemplos notáveis estão relacionados à aproximação do atrito desprezível, seja em problemas envolvendo o atrito de escorregamento, o de rolamento, ou até mesmo o atrito viscoso, quando os estudantes fazem uso inadequado da Equação de Bernoulli.

Na presente análise, fez-se a hipótese de que as parcelas de trabalho $W_{M}, W_{A}$ e $W_{O}$ podem ser modeladas na forma $W=\mu_{R} P \Delta x$ e que $F_{R} \gg F_{A}$ e $F_{R} \gg F_{S}$, de modo que $\mu_{R}$ não dependa da velocidade. Além disso, considerou-se que a dissipação de energia sob a forma de calor devido ao impacto do VANT ao final da rampa poderia ser desprezado. Como se pode perceber, poucas hipóteses foram necessárias devido à simplicidade do problema, assegurando a confiabilidade das medidas realizadas.

Como sugestão para aprimorar o experimento apresentado, acreditamos que seria importante tentar modelar o atrito dos mancais ou rolamentos utilizados, bem como incluir os efeitos aerodinâmicos. Muito importante também seria modelar o efeito do deslizamento lateral sobre o atrito de rolamento, uma vez que este fenômeno é frequentemente observado em decolagens sujeitas a ventos laterais. Por fim, seria muito interessante testar outras configurações de rodas para investigar melhor a dependência um tanto inesperada de $\mu_{R}$ com a carga.

\section{Agradecimentos}

Os autores agradecem à Equipe Trem Ki Voa Micro pelo projeto e construção do protótipo utilizado no estudo e à Universidade Federal de São João del Rei pelo auxílio financeiro que permitiu a sua construção. Agradece ainda aos revisores que, com seus questionamentos sobre o comportamento do coeficiente de atrito sob diferentes cargas, nos permitiu aprofundar e ampliar consideravelmente a análise realizada. 


\section{Referências}

[1] V.L.B. Jesus e D.G.G. Sasaki, Revista Brasileira de Ensino de Física 36, 3503 (2014).

[2] A.V. Andrade-Neto, J.A. Cruz, M.S.R. Miltão e E.S. Ferreira, Revista Brasileira de Ensino de Física 35, 3704 (2013).

[3] N.F.P.L. Santos, Medição Experimental do Coeficiente de Resistência ao Rolamento e do Coeficiente de Arrasto Aerodinâmico de um Veículo Automóvel de Elevada Eficiência Energética. Dissertação de Mestrado, Universidade de Coimbra, Coimbra (2012).

[4] E.V. Costa e C.A.F. Leite, Revista Brasileira de Ensino de Física 32, 4301 (2010).

[5] C.C. Pellegrini e M.S. Rodrigues, Revista Brasileira de Ensino de Física 37, 2307 (2015).

[6] V.L.F. Mossmann, K.B.M.F. Catelli, H. Libardi e I.S. Damo, Revista Brasileira de Ensino de Física 24, 146 (2002).

[7] C.Y. Matuo e J.R. Marinelli, Cad. Bras. Ens. Fis. 24, 132 (2007).

[8] M.A.A. Monteiro, I.C.C. Monteiro e A. Gaspar, Cad. Bras. Ens. Fis. 29, 1121 (2012).

[9] I. Etsion e M. Amit, Journal of tribology 115, 406 (1993).

[10] L.G. Andersen, Rolling Resistance Modelling: From Functional Data Analysis to Asset Management System. Doctoral Thesis, Roskilde University, Roskilde (2015).

[11] F.M. White Mecânica dos fluidos (Editora AMGH, Porto Alegre, 2018), 8 ed.

[12] L.E.M.J. Rodrigues, Fundamentos da Engenharia Aeronáutica (Cengage Learning, Boston, 2013), 1 ed.

[13] B.M. Redrouthu, Tyre modelling for rolling resistance. Masters Dissertation, Chalmers University of Technology, Göteborg (2014).

[14] P. Grover, Modeling of Rolling Resistance Test Data, disponível em https://doi.org/10.4271/980251 (1998) 